\title{
Morfología de Peridiniopsis elpatiewskyi (Ostenfeld) Bourrelly (Dinophyceae) encontrada por primera vez en aguas continentales de Chile
}

\section{Morphology of Peridiniopsis elpatiewskyi (Ostenfeld) Bourrelly (Dinophyceae) found for the first time in Chilean inland waters}

\author{
Enrique Ascencio*, Patricio Rivera \& Fabiola Cruces
}

Departamento de Botánica, Facultad de Ciencias Naturales y Oceanográficas. Universidad de Concepción. Casilla 160-C, Concepción, Chile.

*eascencio@udec.cl; eaascenc@gmail.com

\section{RESUMEN}

\begin{abstract}
Se menciona por primera vez para las aguas continentales de Chile a Peridiniopsis elpatiewskyi (Ostenfeld) Bourrelly, una especie de amplia distribución geográfica, pero escasamente citada para Suramérica. La especie fue encontrada en la laguna Lo Méndez (VIII Región) y observada y fotografiada mediante microscopía fotónica y electrónica de barrido. Su fórmula tabular es Po, X, 4', 7', 6C, $5 \mathrm{~S}, 5^{\prime \prime}, 2$,'". Se describen sus características morfológicas relevantes y se analizan las diferencias entre el material chileno y el descrito en la literatura. Se confirma la preferencia del taxón para aguas eutróficas con pH alcalino.
\end{abstract}

Palabras clave: Dinoflagelados dulceacuícolas, primer registro, distribución geográfica, Chile, Peridiniopsis.

\begin{abstract}
Peridiniopsis elpatiewskyi (Ostenfeld) Bourrelly is reported for the first time from Chilean inland waters, it is a species with a wide geographical distribution but rarely reported from South America. The species was found in Laguna Lo Méndez (VIII Region) and it was observed and recorded under light and scanning electron microscopy. The plate formula is Po, $\mathrm{X}, 4^{\prime}, 7^{\prime \prime}, 6 \mathrm{C}, 5 \mathrm{~S}, 5^{\prime \prime \prime}, 2^{\prime \prime \prime \prime}$. Its main morphological features are described, and the differences between Chilean specimens and those from the literature are discussed. We confirm the preference of this taxon for eutrophic waters with alkaline $\mathrm{pH}$.
\end{abstract}

KEYwoRDs: Freshwater dinoflagellates, first report, geographical distribution, Chile, Peridiniopsis.

\section{INTRODUCCIÓN}

El género Peridiniopsis se creó sobre la base de Peridiniopsis borgei Lemmerman, caracterizada por poseer 6 placas cingulares y una epiteca de 10 placas $\left(4^{\prime}+6^{\prime \prime}\right.$ ó $\left.3^{\prime}+1 a^{2}+6^{\prime \prime}\right)$. La ubicación de las especies de este género ha ido variando según la interpretación de diferentes autores. Lefèvre (1932) las incluye dentro de Peridinium, en cambio, Schiller (1937) las ubica en Glenodinium Ehrenberg. Bourrelly (1968) transfiere a Peridiniopsis las especies de Glenodinium y considera a este último género como mal definido (nomen nudum), ya que la descripción original carece de detalles que permitan diferenciarlo de otros géneros afines. Como resultado de la ampliación del género Peridiniopsis realizada por Bourrelly (1968), se acepta que sus especies poseen una fórmula tabular generalizada $(3-5)^{\prime}-(0-1) \mathrm{a}-(6-8)^{\prime \prime}+5^{\prime \prime \prime}$ $2^{\prime \prime \prime \prime}$, criterio que recibe amplia aceptación (Starmach 1974, Popovský \& Pfiester 1990, Carty 2003). Actualmente el género comprende unas 26 especies mayoritariamente de aguas continentales (Gómez 2012). No obstante, diversas evidencias morfológicas, ultraestructurales y genéticas lo señalan como un género heterogéneo, el cual contiene especies disímiles y con una evidente disparidad en su tabulación (Boltovskoy 1999, Calado \& Moestrup 2002, Takano et al. 2008, Zhang et al. 2014).

El análisis de material proveniente de la laguna Lo Méndez, Concepción, permitió reconocer la presencia de Peridiniopsis, un género que no ha sido anteriormente citado para Chile, representado por Peridiniopsis elpatiewskyi (Ostenfeld) Bourrelly 1968. Esta es una especie ubicua, caracterizada por carecer de placas intercalares y por la presencia de numerosas espinas en la hipoteca. En el presente trabajo se describe a P. elpatiewskyi sobre la base de material chileno, por medio de técnicas de microscopia fotónica y electrónica de barrido.

\section{MATERIALES Y MÉTODOS}

La laguna Lo Méndez $\left(36^{\circ} 48^{`} \mathrm{~S}, 7^{\circ} 02^{`} \mathrm{~W}\right)$ se ubica en el radio urbano de la ciudad de Concepción, Región del 
Biobío, Chile. Es una laguna somera que presenta una fuerte eutroficación antropogénica. El cuerpo de agua posee una forma circular-ovalada, una superficie aproximada de 0,042 $\mathrm{km}^{2}$ y una profundidad máxima de $6,5 \mathrm{~m}$. Esta laguna presenta rangos de temperaturas anuales que fluctúan entre $9,3^{\circ} \mathrm{C}$ y los $23,9^{\circ} \mathrm{C}, \mathrm{pH}$ promedio de 7,9, transparencia (Secchi) 1,51 $\mathrm{m}$, conductividad promedio $281,11 \mu \mathrm{S} / \mathrm{cm}$, rangos de fósforo y nitrógeno total que oscilan entre 40-130 $\mu \mathrm{g} / \mathrm{L}$ y $420-2130$ $\mu \mathrm{g} / \mathrm{L}$ respectivamente (EULA 2013).

El material estudiado fue recolectado entre 25.12.2012 y el 28.01.2013 con una red de abertura de malla de $20 \mu \mathrm{m}$ y se conservó en solución de formaldehido $4 \%$. Más de 50 individuos fueron analizados mediante microscopio fotónico (MO, Olympus CT-31) y electrónico de barrido (MEB, JEOL JSM-6380 LVY). En este último caso, el material fue sometido al secado por punto crítico (Anderson 1951). La terminología y la notación de las placas son las propuestas por Kofoid (1909) y Balech (1974).

\section{RESULTADOS}

\section{Peridiniopsis elpatiewskyi (Ostenfeld) Bourrelly.}

Fig. 1 A-J.

Basiónimo: Peridinium umbonatum Stein var. elpatiewskyi Ostenfeld, Hedwigia 46(6): 391, pl. 9, figs. 9-12. 1907.

Sinónimos: Peridinium elpatiewskyi Lemmermann, Krystogamenflora der Mark Brandenburg, Algen I: 670, figs. 2024. 1910. Peridinium pygmaeum Lindemann, Arch. Naturgesch. 84: 145, figs. 77-80. 1918. Glenodinium elpatiewskyi Schiller, Dinoflagellatae (Peridineae) 10(3)(1): 115, fig. 113 a-f. 1937. Glenodinium pygmaeum Schiller, Dinoflagellatae (Peridineae) 10(3)(1): 114, fig. 111 a-f. 1937. Peridiniopsis elpatiewskyi Bourrelly, Protistologica 4(1): 9. 1968. Peridiniopsis pygmaeum Bourrelly, Protistologica 4(1): 9. 1968.

La longitud de las tecas osciló entre 26,1-38,7 $\mu \mathrm{m}(\mathrm{n}=39)$ y el transdiámetro entre $28,6-31,8 \mu \mathrm{m}(\mathrm{n}=24)$. Las células tienen forma pentagonal (Fig. $1 \mathrm{~A}, \mathrm{~F}) \mathrm{u}$ ovoide con el ápice sobresaliente (Fig. 1 C, G). Las placas presentan crestas marginales de distinto tamaño y espinas en los bordes de las placas de la hipoteca (Fig. 1 F, G). Las aletas cingulares, poco prominentes, sostenidas por dentículos, presentan un aspecto crenulado (Fig. 1 F-I). La superficie de las placas se encuentra ornamentada de nódulos pequeños dispuestos en líneas que conforman un reticulado, además existen nódulos no asociados a las líneas (Fig. 1 E, F, J). Inclusive las espinas de la hipoteca y las crestas suturales poseen nódulos (Fig. 1 E, H). Estas reticulaciones son más marcadas en las placas principales y en individuos más viejos. Los poros presentan ribetes poco elevados y se encuentran distribuidos de forma irregular, adyacentes a las hileras de nódulos (Fig. 1 E-F). Las bandas intercalares (BI), o de crecimiento, presentan diferente grado de desarrollo tanto en distintos ejemplares, como dentro de un mismo individuo, presentan aspecto estriado transversalmente (Fig. 1 B, F, I), debido a hileras paralelas de gruesos nódulos que derivan de las crestas marginales de las placas (Fig. 1 B, F), y longitudinalmente debido a la línea de sutura y además en algunos ejemplares se encontró una estría paralela a la línea de sutura (Fig. $1 \mathrm{~F})$.

La fórmula tabular es Po, X, 4', 7"', 6C, 5S, 5'", $2^{\prime \prime \prime \prime}$. La epiteca es más alta que la hipoteca. Las cuatro placas apicales están en contacto directo con el complejo del poro apical (CPA). Las placas $2^{\prime}, 3^{\prime}, 4^{\prime}$ tocan a la placa del poro (Po), en cambio, la $1^{\prime}$ está en contacto con la placa de la canaleta $(\mathrm{X})$, de posición ventral (Fig. 1 B, D, F). La epiteca no presenta placas intercalares (Fig. 1 B, I). El complejo del poro apical reemplaza totalmente la sutura apical sagital (SAS) (designación de Boltovskoy 1999). La placa del poro apical (Po) es ornamentada por nódulos y presenta un "tapón" (Fig. 1 D-E) que obtura al poro apical de forma circular. Los bordes de la placa presentan crestas marginales que en algunos individuos son bien desarrolladas (Fig. $1 \mathrm{E}$ ), en tanto en otros apenas sobresalen. La placa de la canaleta es de forma rectangular y al igual que Po se encuentra ornamentada por nódulos. El complejo del poro apical está rodeado por una franja continua formada por las crestas marginales de las placas 2',3' y 4' (Fig. 1 E-F, I).

La hipoteca está constituida por 7 placas, cinco postcingulares y dos antapicales. Las dos antapicales son desiguales, siendo la 2"'" de mayor tamaño que la 1"'" (Fig. $1 \mathrm{~J})$. La hipoteca presenta numerosas espinas que nacen de los bordes de las placas $1^{\prime \prime \prime}, 4^{\prime \prime \prime}, 5^{\prime \prime \prime}$ y $1^{\prime \prime \prime \prime}$, pero las más notables en longitud y grosor son las situadas en las zonas en donde la 1"'"' está en contacto con la Sp (Fig. 1 G, J). En nuestro material la segunda placa antapical no presentó espinas (Fig. $1 \mathrm{~J}$ ).

El cingulum es circular o levemente descendente (Fig. 1 C, F) y está formado por 6 placas, donde la $4 \mathrm{C}$ es la más larga y la $\mathrm{C} 1$ es la más corta. La sutura 2"' $/ 3$ "' está alineada con la sutura $3 \mathrm{C} / 4 \mathrm{C}$, la 3 "' $/ 4$ "' puede estar o no alineada con la $4 \mathrm{C} / 5 \mathrm{C}$ (Fig. $1 \mathrm{~A}, \mathrm{G})$ y la $5 \mathrm{C} / 6 \mathrm{C}$ está alineada con la sutura $4 " \prime \prime / 5 "$. Las suturas interprecingulares/intercingulares tienen alineamiento variable, no existiendo una regularidad detectable. El sulcus está formado por 5 placas, penetra levemente en la epiteca y llega hasta el antapex (Fig. 1 C, F, J). La placa sulcal media (Sm) y la sulcal izquierda (Ss) no presentan ornamentaciones. La placa sulcal derecha $(\mathrm{Sd})$ es alargada longitudinalmente y en el lado izquierdo presenta un reborde en forma de aleta no ornamentada que cubre parcialmente a la placa sulcal media y parte de la sulcal izquierda (Fig. 1 C, F). La placa sulcal derecha tiene contacto con la 2"'" mediante su borde posterior (Fig. $1 \mathrm{C}, \mathrm{J}$ ).

\section{DISCUSIÓN}

Las características morfológicas de los individuos encontrados en la laguna Lo Méndez se corresponden totalmente con 

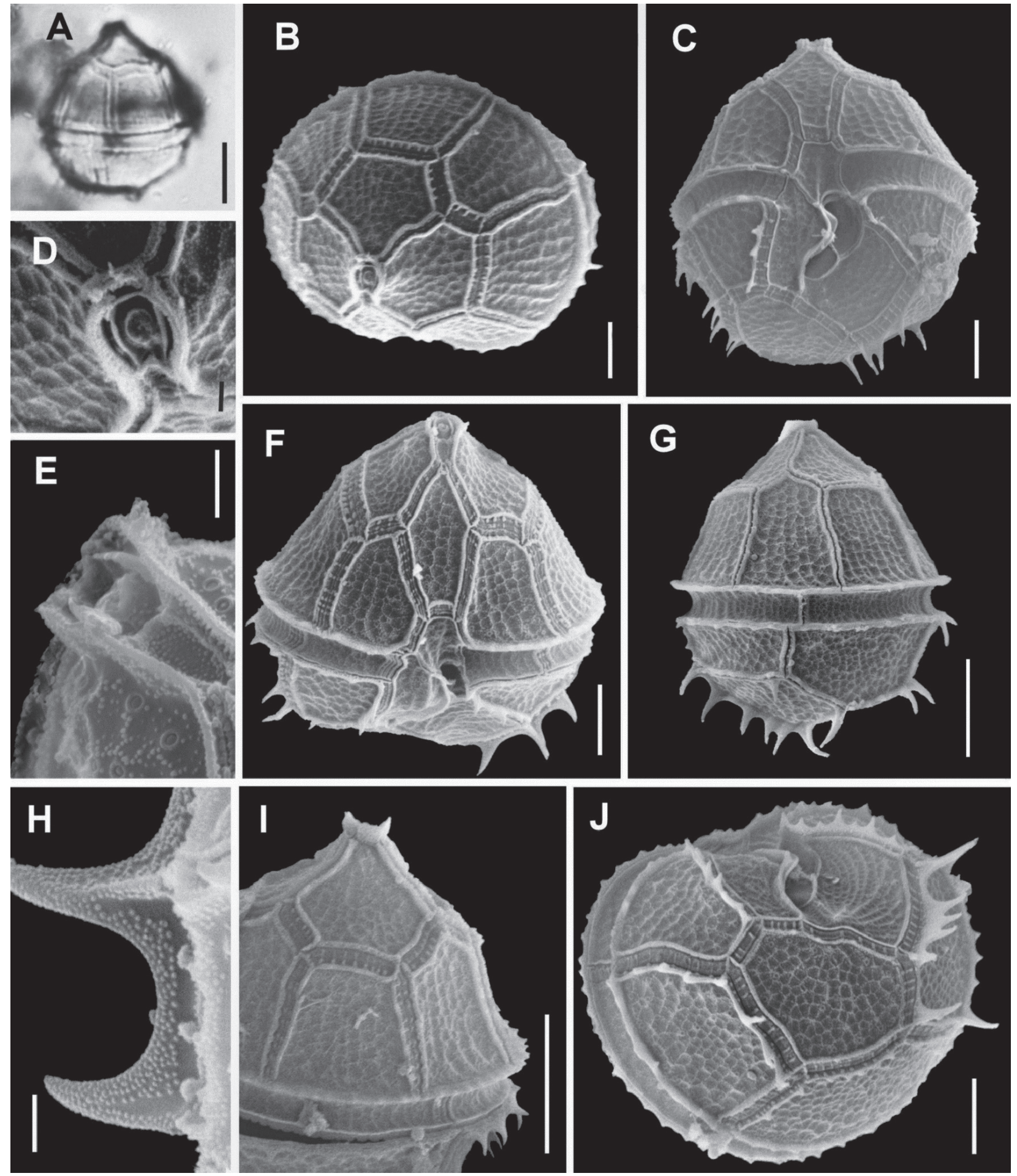

Figura 1: Peridiniopsis elpatiewskyi. A, MO. B-J, MEB. A. Vista dorsal. B. Vista apical. C. Vista ventral con el sulcus amplio que llega al antiapex. D-E. Complejo del poro apical, Po y X presentan nódulos. F. Vista ventral, las bandas de crecimiento presentan gruesos nódulos. G. Vista dorsal, sutura no alineada. H. Detalle de espinas antapicales. I. Vista dorsal. J. Vista antapical. Escalas: A, G, I = $10 \mu \mathrm{m}$; B, C, F, J = $5 \mu \mathrm{m} ; \mathrm{D}, \mathrm{E}, \mathrm{H}=1 \mu \mathrm{m}$.

Figure 1: Peridiniopsis elpatiewskyi. A, ML. B-J, SEM. A. Dorsal view. B. Apical view. C. Ventral view, sulcus widens along reaches the antiapex. D-E. Apical pore complex, Po and X presenting nodules. F. Ventral view, growth bands with thick nodules. G. Dorsal view, suture line displaced. H. Detail of antapical spines. I. Dorsal view. J. Antapical view. Scale bars: A, G, I= $10 \mu \mathrm{m} ; \mathrm{B}, \mathrm{C}, \mathrm{F}, \mathrm{J}=5 \mu \mathrm{m} ; \mathrm{D}, \mathrm{E}, \mathrm{H}=1 \mu \mathrm{m}$. 
aquéllas señaladas por diversos investigadores para distintas localidades del mundo, su forma, medidas y ornamentaciones son poco variables (Ostenfeld 1907, Hansen \& Flaim 2007, Krakhmalny 2009). Nuestros individuos poseen 6 placas cingulares, igual que los ejemplares encontrados por Imamura \& Fukuyo (1990), Bourrelly (1970) y Hansen \& Flaim (2007). Krakhmalny (2009) indica que sus ejemplares estudiados poseen 5 placas cingulares, pero en su lámina I, figura 2, muestra una fotomicrografía de un espécimen en vista ventral en donde marca la placa 6C. P. elpatiewskyi, al igual que lo encontrado por Boltovskoy (1999) en las especies de Glochidinium Boltovskoy tiene la $\mathrm{Sd}$ en contacto con $2^{\prime \prime \prime}$. Esta característica no la posee Peridiniopsis borgei, la especie tipo, y tampoco ninguna especie de Peridinium.

Las bandas intercalares observadas para $P$. elpatiewskyi en este estudio son iguales a las señaladas por Krakhmalny (2009) para la especie, pero difieren significamente de lo mencionado en la literatura para Peridinium s.s., otros Peridiniopsis y Protoperidinium Bergh. En estos géneros las bandas suturales muestran externamente la línea de sutura, pero no las estrías longitudinales. No obstante, Balech (1974) menciona que algunos Protoperidinium eventualmente pueden presentar subdivididas las subfranjas de las bandas. Según Netzel \& Dürr (1984), este carácter se interpretaría como un crecimiento discontinuo. En los ejemplares estudiados las suturas entre las placas postcingulares e intercingulares están alineadas tal como han sido descritas para la especie (Bourrelly 1970, p. 11, figs. 2, 6; Hansen \& Flaim 2007, fig. 23D; Krakhmalny 2009, pl. I, figs. 3-4), exceptuando algunos individuos en donde la sutura 3 "' $/ 4$ "' con la $4 \mathrm{C} / 5 \mathrm{C}$ no está alineada (Fig. $1 \mathrm{G}$ ). Lo anterior evidencia que dicho carácter es variable.

Popovský \& Pfiester (1990) consideran sinónimos a Peridiniopsis elpatiewskyi y Peridiniopsis pygmaeum (Lindemann) Bourrelly debido a su gran semejanza morfológica. Lefèvre (1932) las mantuvo como especies separadas, aunque expresa que ambas sólo se diferencian por sus dimensiones. En monografías clásicas ulteriores también se las considera taxones independientes (Schiller 1937, HuberPestalozzi 1950, Kiselev 1954, Starmach 1974). Popovský \& Pfiester (1990) suponen que Peridinium marchicum Lemmermann var. simplex Woloszyńska es sinónimo de $P$. elpatiewskyi, pero de acuerdo a Meyer \& Elbrächter (1996) son especies diferentes.

Las condiciones tróficas y químicas de la laguna Lo Méndez confirman que $P$. elpatiewskyi es una especie que tiene preferencia por las aguas eutróficas y de $\mathrm{pH}$ levemente alcalino, tal como lo suponen Höll (1928) y Starmach (1974). Nuestros ejemplares fueron revelados durante la temporada de verano, lo que corrobora la preferencia de la especie por aguas cálidas (Hansen \& Flaim 2007, Kiselev 1954, Starmach 1974).

Peridiniopsis elpatiewskyi fue descrita para Mongolia (Ostenfeld 1907) y se ha reportado para diversos lugares de Europa (Hansen \& Flaim 2007, Krakhmalny 2009), Norteamérica (Eddy 1932), Asia (Imamura \& Fukuyo 1990), África (Bourrelly \& Couté 1986) y en Suramérica se ha mencionado para el Amazonas (Uherkovich \& Rai 1979). Parra (2006) en su recopilación bibliográfica de las algas dulceacuícolas de Chile no cita al género. Por lo tanto, este estudio reporta por primera vez al género Peridiniopsis para Chile. Teniendo en cuenta su distribución mundial, la ausencia de registros previos de especies de este género en el país evidencia los escasos estudios taxonómicos realizados en dinoflagelados de sistemas acuáticos continentales.

\section{AGRADECIMIENTOS}

Nuestro reconocimiento a los Dres. A. Boltovskoy y S. Fraga por sus valiosas opiniones durante la realización de este estudio. Al mismo tiempo agradecemos las observaciones y sugerencias de dos revisores anónimos y al personal del Centro de Espectroscopía y Microscopía Electrónica de la Universidad de Concepción (CESMI-UdeC) por la asistencia técnica.

\section{BIBLIOGRAFÍA}

Anderson, T. F. 1951. Techniques for the preservation of three dimensional structure in preparing specimens for electron microscope. Annals of the New York Academy of Sciences, Serie II 13: 130-134.

Balech, E. 1974. El género Protoperidinium Bergh, 1881 (Peridinium Ehrenberg, 1831, partim). Revista del Museo Argentino de Ciencias Naturales "Bernardino Rivadavia", Hidrobiología 4(1): 1-79.

Boltovskoy, A. 1999. The genus Glochidinium gen. nov., with two species: G. penardiforme comb. nov. and G. platygaster $\mathrm{sp.}$ nov. (Peridiniaceae). Grana 38: 98-107.

Bourrelly, P. 1968. Notes sur les Péridiniens d'eau douce. Protistologica 4: 5-16.

Bourrelly, P. 1970. Les algues d'eau douce. Tome III: Les algues bleues et rouges. Les eugléniens, peridiniens et crytomonadines. Éditions N. Boubée \& Cie. Paris. 512 pp.

Bourrelly, P. \& A. Couté. 1986. Algues d'eau douce de 1'lle Maurice (Diatomées exclues). Revue d'Hydrobiologie Tropicale 19(2): 131-158.

Calado, A. J. \& Ø. Moestrup. 2002. Ultrastructural study of type species of Peridiniopsis, Peridiniopsis borgei (Dinophyceae), with special reference to the peduncle and flagellar apparatus. Phycologia 41(6): 567-584.

CARTY, S. 2003. Dinoflagellates. In: D.L. Wehr \& R.G. Sheath (eds.), Freshwater algae of North America ecology and classification, pp. 685-714. Academic Press, New York.

EDDY, S. 1932. The fresh-water armored or thecate dinoflagellates. Transactions of American Microscopical Society 49(4): 277-321.

EULA. 2013. Agua, situaciones de emergencia, factibilidad técnica para el abastecimiento de agua potable desde un sistema de 
lagunas urbanas [InnovaChile, Proyecto 10CREC-8453]. $335 \mathrm{pp}$.

GómEz, F. 2012. A checklist and classification of living dinoflagellates (Dinoflagellata, Alveolata). CICIMAR Oceánides 27(1): 65140.

Hansen, G. \& G. Flaim. 2007. Dinoflagellates of the Trentino Province, Italy. Journal of Limnology 66(2): 107-141.

HöLl, K. 1928. Oekologie der Peridineen. Pflanzenforschung 11: 1-105.

Huber Pestalozzi, G. 1950. Das Phytoplankton des Süßwassers. Crytophyceen, Chloromonadinen, Peridineen. In: A. Thienemann (ed.), Die Biennengewässer. 16(3), 310 pp. E. Schweizerbart'sche Verlagsbuchhandlung, Stuttgart.

ImamuRa, K. \& Y. FukuYo. 1990. Peridinium elpatiewskyi (Ostenfeld) Lemmermann. In: Y. Fukuyo, H. Takano, M. Chihara \& K. Matsuoka (eds.), Red tide organisms in Japan- An illustrated taxonomic guide, pp. 126-127. Uchida Rakakuho, Tokio.

Kiselev, I. A. 1954. Pyrrophyte algae. Manual for identification of freshwater algae of the USSR. Vol 6. Sovetskaya Nauka, Moscow. 212 pp (In Russian).

KofoID, C. A. 1909. On Peridinium steinii Jörgensen, with a note on the nomenclature of the skeleton of Peridinidae. Archiv für Protistenkunde 16: 25-47, 2 pl.

Krakhmalny, A. F. 2009. Morphology of Peridiniopsis elpatiewskyi (Ostenf.) Bourr. (Dinophyta) theca. International Journal on Algae 11(1): 25-33.

LefĖvre, M. 1932. Monographie des espèces d'eau douce du genre Peridinium Ehrb. Archives de Botanique 2(5):1-210, 6 pl.

Meyer, B. \& M. Elbrächter. 1996. Proposal to conserve the name Peridinium elpatiewskyi (Dinophyceae) with a conserved type. Taxon 45: 531-532.
Netzel, H. \& G. Dürr. 1984. Dinoflagellate cell cortex. In: D. L. Spector (ed.), Dinoflagellates, pp 43-105. Academic Press, Florida.

Ostenfeld, C.H. 1907. Beiträge zur Kenntnis der Algenflora des Kossogol-Beckens in der nordwestlichen Mongolei, mit spezieller Berücksichtingung des Phytoplanktons. Hedwigia 46: 365-420.

PARRA, O. 2006. Estado de conocimiento de las algas dulceacuícolas de Chile (excepto Bacillariophyceae). Gayana 70(1): 8-15.

Popovský, J. \& L. A. Pfiester. 1990. Dinophyceae (Dinoflagellida). In: H. Ettl, J. Gerloff, H. Heynig \& D. Mollenhauer. (eds.), Süßwasserflora von Mitteleuropa Vol. 6, 272 pp. Gustav Fischer Verlag, Jena.

SChILler, J. 1937. Dinoflagellatae (Peridineae) in monographischer behandlung. In R. Kolkwitz (ed.), Rabenhorst's KrytogamenFlora von Deutschland, Österreich und der Schweiz. Vol. 10(3), 589 pp. Akademische Verlagsgesellschaft, Leipzig.

Starmach, K. 1974. Crytophyceae, Dinophyceae, Raphidophyceae. In: K. Starmach \& J. Siemińska (eds.), Flora Słodkowodna Polski. Vol. 4, 520 pp. Państwowe Wydawnictwo Naukowe, Warszawa, Kraków.

Takano, Y., G. Hansen, D. Fujta \& T. Horiguchi. 2008. Serial replacement of diatom endosymbionts in two freshwater dinoflagellates, Peridiniopsis spp. (Peridiniales, Dinophyceae). Phycologia 47(1): 41-53.

Uherkovic, G. \& H. Rai. 1979. Algen aus dem Rio Negro und seinen Nebenflüssen. Amazoniana 6(4): 611-638.

Zhang, Q., G. Liu \& Z. Hu. 2014. Description of a new freshwater bloom-forming dinoflagellate with a diatom endosymbiont, Peridiniopsis minima sp. nov. (Peridiniales, Dinophyceae) from China. Algological Studies 145/146: 119-133.

Recibido: 05.09.14

Aceptado: 19.03 .15 